\title{
Broadband Polarimetry with the Square Kilometre Array: A Unique Astrophysical Probe
}

B. M. Gaensler ${ }^{* \neq 1+1,2}$, Iván Agudo ${ }^{3}$, Takuya Akahori ${ }^{1,4}$, Julie Banfield ${ }^{5,6}$, Rainer Beck ${ }^{7}$, Ettore Carretti $^{5}$, Jamie Farnes ${ }^{1,2}$, Marijke Haverkorn ${ }^{8}$, George Heald ${ }^{9}$, David Jones ${ }^{8}$, Thomas Landecker ${ }^{10}$, Sui Ann Mao ${ }^{7}$, Ray Norris ${ }^{5,2}$, Shane O'Sullivan ${ }^{1,2,11}$, Lawrence Rudnick $^{12}$, Dominic Schnitzeler ${ }^{7}$, Nicholas Seymour ${ }^{5,13}$, and Xiaohui Sun ${ }^{1}$

${ }^{1}$ The University of Sydney; ${ }^{2}$ ARC Centre of Excellence for All-sky Astrophysics (CAASTRO);

${ }^{3}$ JIVE; ${ }^{4}$ Kagoshima University; ${ }^{5}$ CSIRO; ${ }^{6} A N U ;{ }^{7}$ MPIfR; ${ }^{8}$ Radboud Universe Nijmegen;

${ }^{9}$ ASTRON; ${ }^{10}$ DRAO; ${ }^{11}$ UNAM; ${ }^{12}$ University of Minnesota; ${ }^{13}$ ICRAR - Curtin University

E-mail: bryan.gaensleresydney •edu.au

Faraday rotation of polarised background sources is a unique probe of astrophysical magnetic fields in a diverse range of foreground objects. However, to understand the properties of the polarised sources themselves and of depolarising phenomena along the line of sight, we need to complement Faraday rotation data with polarisation observations over very broad bandwidths. Just as it is impossible to properly image a complex source with limited $u$ - $v$ coverage, we can only meaningfully understand the magneto-ionic properties of polarised sources if we have excellent coverage in $\lambda^{2}$-space. We here propose a set of broadband polarisation surveys with both SKA1 and SKA2, which will provide a singular set of scientific insights on the ways in which galaxies and their environments have evolved over cosmic time.

Advancing Astrophysics with the Square Kilometre Array

June 8-13, 2014

Giardini Naxos, Italy

\footnotetext{
*Speaker.

$\dagger$ On behalf of the SKA Cosmic Magnetism Working Group

$\ddagger$ Present address: Dunlap Institute for Astronomy and Astrophysics, The University of Toronto
} 


\section{Introduction}

Faraday rotation is a superb probe of astrophysical magnetic fields at all redshifts. This point was made strongly in the 2004 Square Kilometre Array (SKA) Science Case, in which Beck \& Gaensler (2004) proposed a 1.4-GHz polarisation survey with the SKA covering $10000 \mathrm{deg}^{2}$, resulting in a closely spaced "Faraday rotation measure grid" of active galactic nuclei (AGN). As discussed by Gaensler et al. (2004), this rotation measure (RM) grid could then be used to study the detailed magneto-ionic properties of the Milky Way, nearby galaxies, distant galaxies and galaxy clusters, with the ultimate goal of distinguishing between different origins for magnetism throughout the Universe. "Cosmic Magnetism" was correspondingly designated one of the five Key Science Projects for the SKA (Gaensler 2004; Carilli \& Rawlings 2004).

A decade later, the relevance and importance of the RM grid have only increased (see Taylor et al. 2007, 2009; Stepanov et al. 2008; Krause et al. 2009; Grant et al. 2010; Hammond et al. 2014; Oppermann et al. 2012, 2014; Hales et al. 2014a; Rudnick \& Owen 2014; Stil et al. 2014; Akahori et al. 2014). Specifically, the RM grid remains a powerful tool for probing foreground magnetic fields, i.e., cases where the polarised emitting region and Faraday rotating medium are distinct and well separated, and where the combined statistical properties of a large number of sightlines can be used to extract the global magneto-ionic properties of intervening material (Johnston-Hollitt et al. 2015).

\subsection{Beyond The Rotation Measure Grid}

For cases in which the polarised emission and Faraday rotation occur in the same source, or in which we are studying the polarisation properties of a single sightline rather than the large ensemble probed by the RM grid, our understanding of polarisation and Faraday rotation has substantially deepened and matured over the last ten years. For example, Beck \& Gaensler (2004) assumed that around $50 \%$ of polarised AGN would suffer from internal depolarisation, which would manifest itself as a non-linear dependence of polarisation position angle, $\theta$ on $\lambda^{2}$ (where $\lambda$ is the observing wavelength). However, we now realise that even quite simple scenarios (e.g., a source consisting of two spatially unresolved polarised components, each with different RMs) can produce an apparent linear relationship between $\theta$ and $\lambda^{2}$, but corresponding to a spurious value of RM; only when one considers the fractional polarisation, $\Pi \equiv \sqrt{\left(Q^{2}+U^{2}\right)} / I$, as a function of $\lambda^{2}$ does the observer realise that something is amiss (Farnsworth et al. 2011). In such cases, and also in sources for which there is no linear dependence of $\theta$ on $\lambda^{2}$, we also now better appreciate the various ways in which $q \equiv Q / I, u \equiv U / I$ and $\Pi$, all as a function of $\lambda^{2}$, can provide detailed information on magnetic fields, ionised gas and turbulence (Bell et al. 2011; Farnsworth et al. 2011; Beck et al. 2012; Bernet et al. 2012; Farnes et al. 2014a; Brandenburg \& Stepanov 2014; Horellou \& Fletcher 2014). Furthermore, it is now clear that over a fractional bandwidth of $25 \%$ as assumed by Beck $\&$ Gaensler (2004), there are considerable degeneracies as to the nature of the observed Faraday rotation (Law et al. 2011; O’Sullivan et al. 2012). Finally, while Beck \& Gaensler (2004) proposed that fitting $\theta$ vs $\lambda^{2}$ or applying RM synthesis Brentjens \& de Bruyn (2005) should be sufficient to extract Faraday rotation in most cases, we now realise that a measurement of RM and its error for a polarised source is an extremely complex problem that as yet has no optimal solution (e.g., 
Farnsworth et al. 2011; Hales et al. 2012; Macquart et al. 2012; Andrecut et al. 2012; Ideguchi et al. 2014; Kumazaki et al. 2014; Sun et al. 2014).
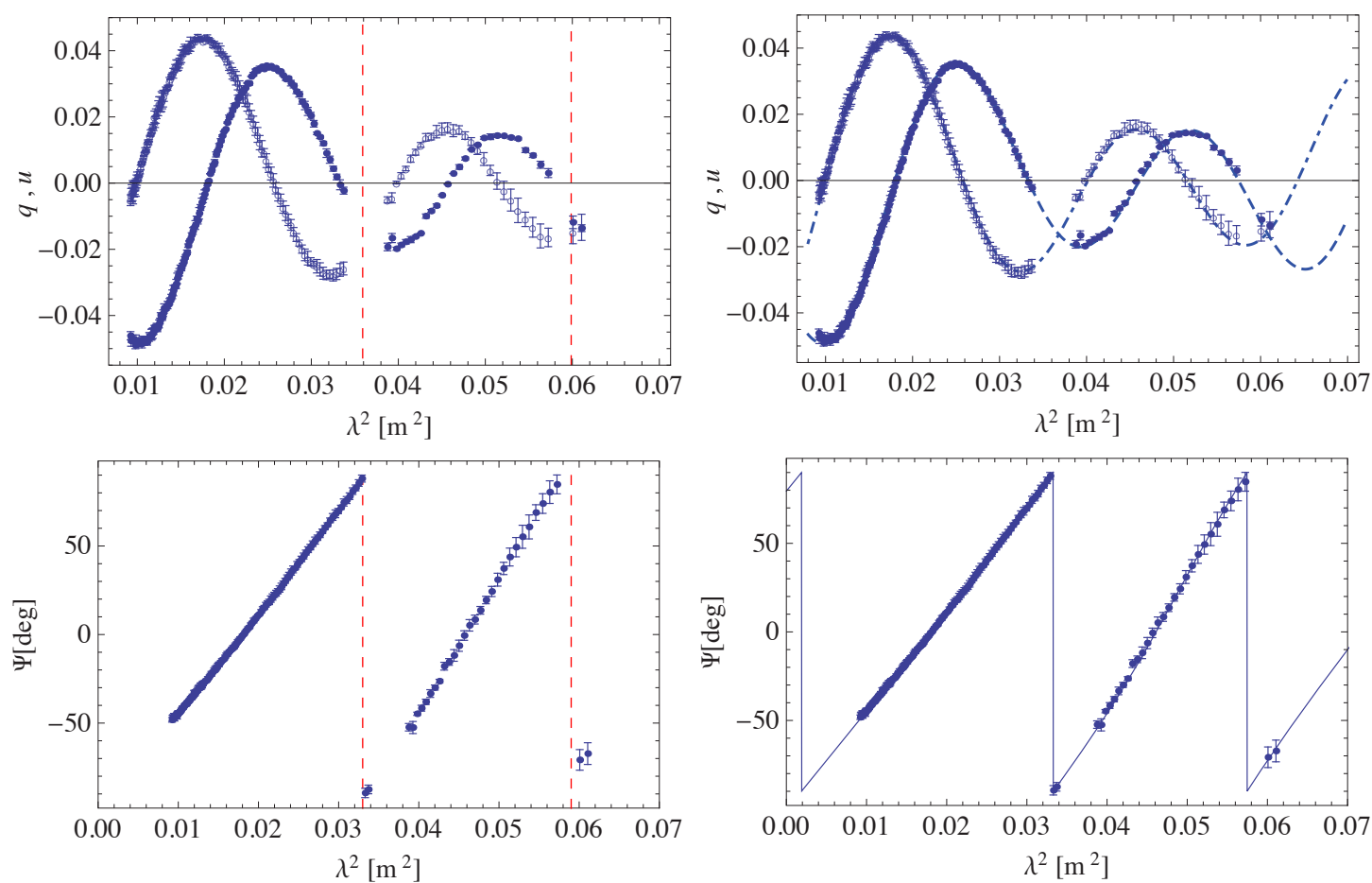

Figure 1: Polarisation data for PKS B1610-771 over the frequency range 1.1 to $3.1 \mathrm{GHz}$ (adapted from Figure 12 of O'Sullivan et al. 2012). Top panel: $q$ (open circles) and $u$ (filled circles) vs $\lambda^{2}$. On the left-hand side, the vertical red lines show the range in $\lambda^{2}$ corresponding to an observation of bandwidth $350 \mathrm{MHz}$ centred on $1.4 \mathrm{GHz}$; on the right-hand side, the data are fit over the full bandwidth to a model containing two RM components. Bottom row: as for the top row, but showing polarisation angle vs $\lambda^{2}$.

Some of these issues are exemplified in Figure 1, which shows an observation by O'Sullivan et al. (2012) of the bright polarised quasar PKS B1610-771 over the frequency range 1.1 to 3.1 GHz. Three features of note are apparent from these data:

1. The plot of polarisation angle vs $\lambda^{2}$ deviates from a purely linear dependence;

2. The plots of $q$ and $u$ vs $\lambda^{2}$ do not show sinusoids of constant amplitude, and $\Pi$ is not constant as a function of $\lambda^{2}$;

3. If observed over a relatively narrow bandwidth, none of these behaviours would be apparent.

For example, Beck \& Gaensler (2004) consider a representative SKA survey with a bandwidth of $350 \mathrm{MHz}$ centred on $1.4 \mathrm{GHz}$, corresponding to the vertical red lines in the left column of Figure 1 . With such observations, a user would infer a single foreground RM value of $+135 \mathrm{rad} \mathrm{m}^{-2}$, would not realise that this value was likely in error, and would have no capacity to identify or understand this source's potentially interesting properties. In contrast, consider the right column of 
Figure 1, which shows the same data but now fit with a simple model consisting of two spatially resolved components with different RMs. This shows an excellent match to the data, and allowed O'Sullivan et al. (2012) to infer that this source most likely contains two polarised knots with RMs ${ }^{1}$ of $+107 \mathrm{rad} \mathrm{m}^{-2}$ and $+79 \mathrm{rad} \mathrm{m}^{-2}$.

\subsection{Broadband Polarimetry with the SKA}

Beyond the simple example described in $\S 1.1$, there are a whole range of complex behaviours that can only be identified and distinguished if radio polarisation data are recorded over sufficient bandwidth and at high angular resolution and sensitivity. Just as it is impossible to properly image a complex source with limited $u$ - $v$ coverage, we can only meaningfully understand the magneto-ionic properties of the polarised sources themselves if we have excellent coverage in $\lambda^{2}$-space.

In this Chapter, we highlight the unique new physical insights provided by SKA polarisation surveys over a wide contiguous frequency range. It is important to emphasise that these broadband experiments complement the RM grid, rather than supersede it. The purpose of the RM grid is to study magnetic fields in extended foreground sources such as the Milky Way and the intergalactic medium (IGM). ${ }^{2}$ In contrast, broadband data either provide detailed information on individual sightlines, or can better probe the intrinsic properties of the polarised emitters and their environments. The RM grid primarily probes Faraday rotation, while the broadband studies proposed here primarily probe depolarisation. More fundamentally, broadband polarimetry transcends the study solely of magnetic fields, and instead becomes a highly sensitive tool for addressing a much broader set of issues. In the sections below, we demonstrate that wide-area wide-band polarimetric surveys with the SKA will provide us with powerful new data sets aimed at addressing the following questions:

- What is the relationship between supermassive black holes and their environments? (§2)

- What are the physical properties of absorbing systems? (\$3)

- How have galaxies evolved over cosmic time? (\$4)

As will be explained below, $\S 2$ and $\S 4$ focus on polarisation as a probe of the polarised sources themselves and of their immediate environments, while $\S 3$ considers the use of background sources to study the effects of Faraday rotation and depolarisation in intervening objects along the line of sight. In $\$ 5$ we quantify the expected science outcomes for SKA1 and note the relevant observing specifications. In $\S 6$, we consider an early science program for SKA1 once it reaches $50 \%$ sensitivity. In $§ 7$, we anticipate the possible science outcomes that can be pursued with SKA2.

\section{What is the Relationship Between Supermassive Black Holes and Their Environments?}

All massive galaxies host supermassive black holes (SMBHs). Relativistic outflows from these SMBHs deposit enormous amounts of mechanical energy into their surroundings, pollute the IGM

\footnotetext{
${ }^{1}$ Note that $\mathrm{RM}=+135 \mathrm{rad} \mathrm{m}^{-2}$ (as would be inferred from observations over a narrow bandwidth) is not even in the range bracketed by these two values.

${ }^{2}$ Sources such as that shown in Figure 1 do not invalidate the RM-grid approach, because only $\lesssim 25 \%$ of polarised sources are expected to show behaviour that deviates from the idealised "Faraday thin" case (Law et al. 2011). In addition, the wideband data described here will allow us to explicitly exclude such sources from the RM grid.
} 
with metals and magnetic fields, and regulate star-formation and feedback. A key issue underpinning all these considerations is the extent to which thermal gas from the host galaxy and its surroundings interacts with and is entrained by these outflows. Such entrainment can decelerate the AGN's relativistic jets, suppress star formation, regulate the growth of the central SMBH, and control the acceleration efficiency of ultra-high-energy cosmic rays

Detailed polarimetric studies of individual sources have begun to reveal some of the complex ways in which radio lobes from AGN can interact with their environments - for some sources we see thermal sheaths draped over the radio lobes (e.g., Guidetti et al. 2012), in others we observe compression and mixing of the surrounding thermal gas (e.g., Guidetti et al. 2011), and at least in one case we appear to detect thermal gas inside the lobes (O'Sullivan et al. 2013).

However, for the broader population we lack meaningful sample sizes, spatially resolved spectropolarimetry, and a surrounding RM grid to correct for foreground Galactic Faraday rotation. With the SKA, we have the capacity to comprehensively explore the distribution of thermal gas in and around radio lobes from AGN. By simultaneously measuring polarised fractions, Faraday rotation and synchrotron intensity across a broad bandwidth, with the high angular resolution needed to spatially resolve lobes and distinguish them from cores and host galaxies, and in conjunction with information on foreground and background Faraday rotation as supplied by the RM grid, we can provide a comprehensive view of entrainment, outflows, ionised gas and magnetic fields in radio galaxies, covering a wide range of host galaxies, jet powers, environments and redshifts.

\section{What Are The Physical Properties of Absorbing Systems?}

A fundamental limitation in radio astronomy is the difficulty in studying normal (star-forming) galaxies at large distances. While the SKA will provide the enhanced sensitivity needed to see such sources out to higher redshifts than currently possible, a powerful alternative approach is to study otherwise invisible populations in projection against bright background sources. For the SKA this is typified by surveys for $\mathrm{H}$ I absorption toward distant AGN, which will allow an unbiased study of the evolution of normal galaxies over a huge range of cosmic time (Kanekar \& Briggs 2004; Morganti et al. 2015).

Polarimetry and Faraday rotation provide an equivalent probe of unseen intervenors, but for ionised rather than neutral gas. Specifically, the detailed magneto-ionic properties of normal galaxies can be studied out to high redshift by determining RMs and polarised fractions as a function of redshift, and by cross-correlating this information with $\mathrm{Mg}$ II spectroscopy and deep optical imaging (Kronberg et al. 2008; Bernet et al. 2012, 2013; Farnes et al. 2014b). Through this technique, we can potentially measure the amplitude of turbulence in galactic disks and halos, the amplification time scales and coherence lengths of galactic dynamos, and the covering fraction and spatial extent of intervening systems, all as a function of redshift. However, a number of technical limitations currently prevent any meaningful progress in these areas. To advance this topic, we require:

- Broad bandwidths, with which we can break the degeneracy between different types of Faraday rotation and depolarisation along the line of sight;

- High sensitivity and survey speed, through which we can accumulate a meaningful sample of sources that have both radio polarisation and optical spectroscopy data; 
- High angular resolution, with which we can isolate the same sightlines in radio polarisation as are probed by the corresponding optical spectra.

The SKA fulfills all these criteria. When combined with $\mathrm{H}$ I absorption surveys (which perhaps can be performed commensally) plus data from next-generation optical and infrared facilities such as 4MOST, WFIRST, LSST and Euclid, we can obtain a complete view of the gas, turbulence, magnetisation and spatial extents of ordinary galaxies over a wide range of redshifts.

\section{How Have Galaxies Evolved Over Cosmic Time?}

The experiment described in $\S 2$ is one of many measurements in which we wish to study the intrinsic properties of radio sources, and to determine how radio galaxies and their associated SMBHs and host galaxies have evolved as a function of redshift. However, any comparison of such sources at different redshifts will be meaningless unless the observed properties are first corrected into the frame of reference in which the emission or Faraday rotation occurs. Usually this requires the application of a "K-correction" (e.g., Hogg et al. 2012), in which the data are shifted to a higher frequency than that with which they were observed. For many applications, the K-correction is simply a direct extrapolation of the source's observed flux using the redshift and spectral index. However, for polarimetry the situation is more complex. Polarisation from AGN as a function of wavelength can show a wide variety of behaviours: some sources depolarise, others "repolarise", and others show oscillatory or other behaviour, as shown in Figure 2. These phenomena are further compounded when a polarised source is spatially unresolved, and contains multiple components with different spectral indices and different polarised fractions.

Thus to determine the polarisation properties of a source in its rest frame, one must directly observe the emitted and subsequently redshifted radiation - extrapolation to frequencies outside the observing band is generally not possible. Such studies require near-continuous polarisation data observed or interpolated across a wide range of frequencies. The wide band is needed both to compare sources over a range of redshifts at a single fixed emitting wavelength, and to characterise the polarisation spectral energy distributions (SEDs) of individual sources. Farnes et al. (2014a) have presented the first large catalogue of polarisation SEDs suitable for comparison of objects at different redshifts in a common rest frame, consisting of 951 sources with multiple polarisation measurements over the frequency range $0.4-100 \mathrm{GHz}$ (see examples in Fig. 2). While the frequency range covered is very large, the sampling is sparse and inconsistent, the individual data points come from different surveys taken at different epochs with different telescopes and differing angular resolutions, and the overall sample size is small.

Using the SKA, broadband polarimetry can provide a very large sample of polarisation SEDs, suitable for robust and accurate rest-frame corrections for many different types of sources over a wide range of redshifts. ${ }^{3}$ This will enable a diverse set of powerful experiments with which we can study the polarisation properties of radio galaxies and AGN as a function of redshift. In addition,

\footnotetext{
${ }^{3}$ Note that two sources at different redshifts will have different effective spacings of adjacent frequency channels in their rest frames. This will mean that a large RM seen at one redshift might not be detectable at another redshift, as per Equation (63) of Brentjens \& de Bruyn (2005). However, in practice the expected channel widths for the SKA will be sufficiently narrow that all physically plausible values of RM will be detectable at all redshifts.
} 

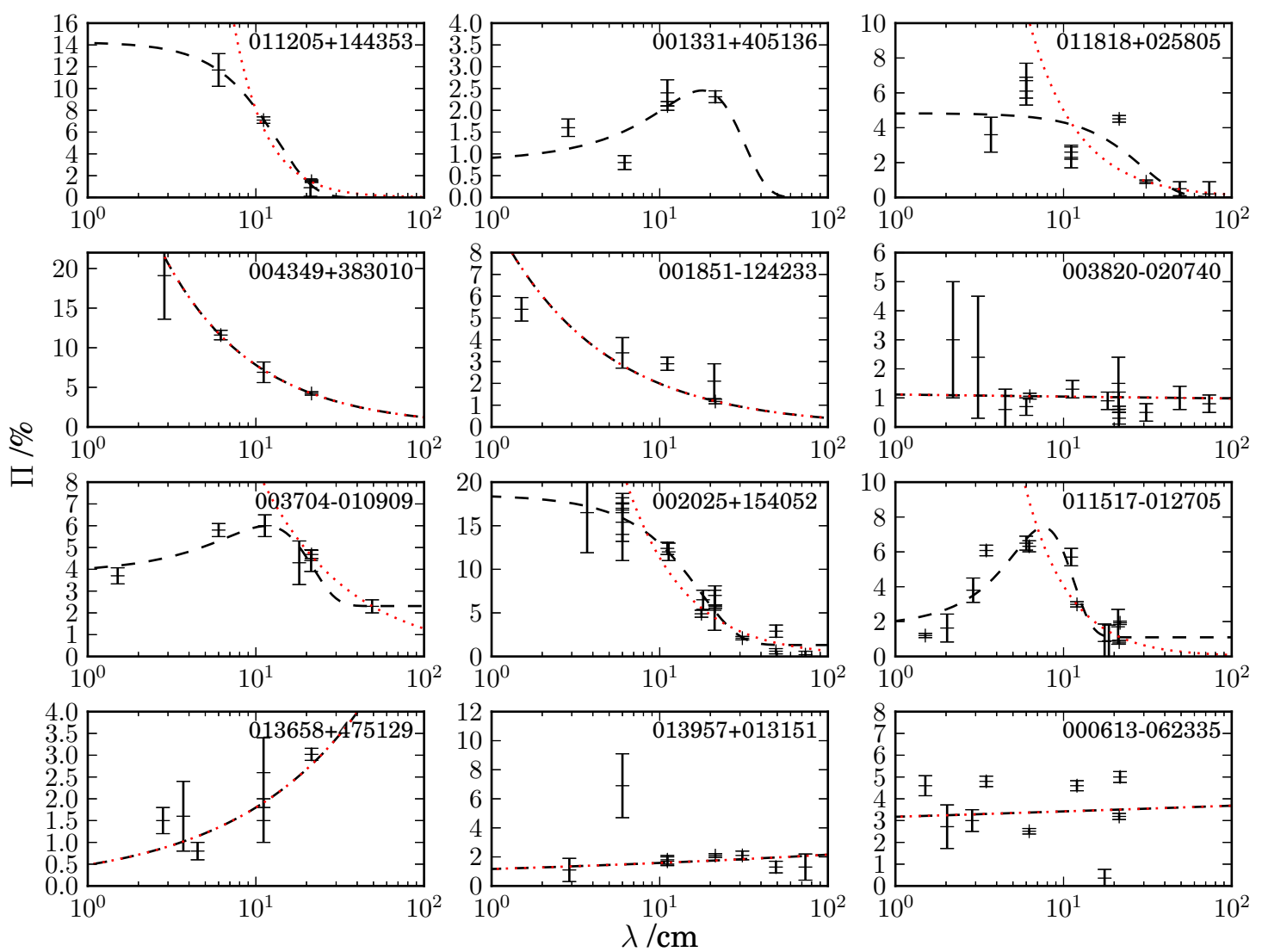

Figure 2: $\Pi$ vs $\lambda$ for 12 sources, reproduced from Farnes et al. (2014a) by permission of the AAS. The best-fit polarisation SED chosen from four simple models is shown as a black dashed line: the top row shows sources whose best-fitting polarisation SED has a Gaussian profile, the second row shows depolarising sources, the third row shows SEDs modelled by a Gaussian with a constant term and the last row shows repolarising sources. The red dotted line shows the best-fit power law for $\Pi$ vs $\lambda$. The quality of the fits decreases from left to right.

the SKA will simultaneously obtain total intensity SEDs for an even larger number of sources, which will facilitate detailed studies of spectral indices, spectral curvature and spectral turnover.

\section{Science Outcomes for SKA1}

The experiments proposed above all require sensitive high-resolution polarisation observations of a large sample of sources over a broad bandwidth. Both SKA1-MID and SKA1-SUR meet these basic specifications. For the RM grid experiment, two additional requirements were all-sky coverage (to fully probe the magnetic field of different parts of the Milky Way) and a very dense sampling of polarised sources (to provide multiple closely spaced sightlines through extended foreground sources). The first of these considerations is not relevant for our purposes, and the second is of reduced importance. Instead, our main drivers are to optimise the frequency coverage and to maximise the number of sources detected. A full consideration of all survey specifications is 
beyond the scope of this Chapter, but as for other proposed polarisation and continuum surveys, consideration needs to be given to the requirements on dynamic range, flux calibration (both relative and absolute), $u-v$ coverage, mosaicing strategy and wide-field polarisation purity.

\subsection{Choice of Observing Frequency}

Arshakian \& Beck (2011) have considered the optimum observing frequency, $\nu_{\mathrm{opt}}$, for the study of magnetic fields. They define $\nu_{\mathrm{opt}}$ as the frequency at which the polarised intensity is a maximum, while requiring a broad bandwidth around this frequency to distinguish between different Faraday and depolarisation effects. Within a given source or intervenor population, at a redshift $z$ and for a standard deviation $\sigma_{\mathrm{RM}}$ in RM or Faraday depth (in the frame of the Faraday rotating medium), we have that $\nu_{\mathrm{opt}} \propto \sigma_{\mathrm{RM}}^{1 / 2}(1+z)^{-1}$. For the case of entrained thermal gas in and around the lobes of radio galaxies as considered in $\S 2$, we adopt $\sigma_{\mathrm{RM}} \sim 3-6 \mathrm{rad} \mathrm{m}^{-2}$ (O'Sullivan et al. 2013), for which we infer $\nu_{\mathrm{opt}} \approx 900-1300(1+z)^{-1} \mathrm{MHz}$. For intervening galaxies along the line of sight as discussed in $\S 3$, we adopt the "faint galaxy halo" case of Arshakian \& Beck (2011), for which $|\mathrm{RM}| \approx \sigma_{\mathrm{RM}} \approx 8 \mathrm{rad} \mathrm{m}^{-2}$ and $\nu_{\mathrm{opt}} \approx 1200-1500(1+z)^{-1}$ MHz. Finally, for corrections for redshift as proposed in $\S 4$, we wish to maximise the redshift range over which sources within a given population can be compared. To achieve this, we must observe over as broad a band as possible, encompassing the optimal frequency $\nu_{\text {opt }}$. SKA1-MID Band 2 (950-1760 MHz) and SKA1-SUR Band $2(650-1670 \mathrm{MHz})$ both provide the required frequency coverage. SKA1-SUR is preferred because it extends down to lower frequencies than SKA1-MID: in $\lambda^{2}$-space, the coverage of Band 2 is 2.5 times larger for SKA1-SUR than for SKA1-MID. The wavelength coverage of SKA1-MID could be extended by additionally observing with SKA1-MID Band 1 (350-1050 MHz), but the total observing time required would be larger than for SKA1-SUR, despite the larger instantaneous bandwidth for SKA1-MID.

In subsequent discussion, we consider a broadband continuum polarisation survey using SKA1SUR $2(650-1670 \mathrm{MHz}){ }^{4}$ This optimises the study of radio lobes (\$2) for emitting sources in the redshift range $z \lesssim 0.4-1$ and for intervenors (\$3) in the range $z \lesssim 0.8-1.3$, while allowing corrections into a common rest frame ( $\$ 4)$ for a population covering the redshift range $\Delta z \approx 1.6\left(1+z_{\mathrm{min}}\right)$ for a minimum redshift $z_{\min }$. Thus all three topics can be simultaneously pursued for sources or intervenors in the redshift range $0<z<1$.

\subsection{Choice of Angular Resolution}

The experiments described above require angular resolution sufficiently high to spatially resolve the polarised emission from radio lobes (§2), to match polarisation information to the locations of optical spectra ( $\$ 3)$, and to resolve the polarised emission into multiple components $(\S 4)$. Angular resolutions of $\sim 10^{\prime \prime}$ to $\sim 1^{\prime}$, while suitable for the RM grid, cannot address the questions envisaged here.

Our knowledge of polarised morphologies for the faint radio sky is limited. The two deepest polarimetric observations are those of Hales et al. (2014a,b) and Rudnick \& Owen (2014). Hales et al. $(2014 \mathrm{a}, \mathrm{b})$ presented observations at $10^{\prime \prime}$ resolution, covering the frequency range $1.3-1.5 \mathrm{GHz}$ to a sensitivity of $\approx 30 \mu \mathrm{Jy}_{\text {beam }}{ }^{-1}$. Rudnick \& Owen (2014) have reported observations at 1 ."6 $^{\prime \prime}$

\footnotetext{
${ }^{4}$ We note that a possible shift of SKA1-SUR Band 2 to $560-1430 \mathrm{MHz}$ is currently under consideration.
} 
resolution, covering the frequency range $1.3-1.9 \mathrm{GHz}$ to a sensitivity of $\approx 2.4 \mu \mathrm{Jy}_{\text {beam }}{ }^{-1}$. At $10^{\prime \prime}$ resolution, $36 \%$ of polarised sources show extended structure or multiple components in polarised intensity (Hales et al. 2014a), while at 1."6 resolution, 69\% of polarised sources show spatial extent in polarisation ${ }^{5}$ (Rudnick \& Owen 2014). Thus arcsecond-level (or better) resolution is desired, so as to maximise the number of extended polarised sources detected. This choice also provides the angular resolution needed to compare sources to optical data, and avoids confusion in total intensity even for relatively long integrations. In summary, the angular resolution of the full SKA1-SUR array $\left(1^{\prime \prime}\right.$ at $\left.1.4 \mathrm{GHz}\right)$ is a good match to our requirements.

\subsection{Survey Specifications}

We first consider the science requirements to study the interaction of radio lobes with thermal gas (§2). To fully investigate this phenomenon, we need a sample large enough to group such sources into 10 redshift bins, 10 luminosity bins and 10 morphological categories (see $\$ 6.2 .2$ of Hales et al. 2014b), with 100 sources in each of these 1000 subsets. We thus aim to observe a total of $10^{5}$ polarised, extended, radio galaxies. Assuming that $\sim 70 \%$ of polarised sources are spatially extended at arcsec-resolution (Rudnick \& Owen 2014) and that $\sim 20 \%$ of sources show complex Faraday depth spectra that might be the signature of interactions with thermal gas, we must observe $7 \times 10^{5}$ polarised sources to obtain our required data set, or a sky density of $\sim 23$ sources $/ \mathrm{deg}^{2}$ for a survey covering $30000 \mathrm{deg}^{2}$. This sky density is reached for a peak polarised intensity (at $1.4 \mathrm{GHz}$ and at $1 . " 6$ resolution) of $\approx 0.1 \mathrm{mJy}^{\text {beam }}{ }^{-1}$ (Rudnick \& Owen 2014). The signal-to-noise ratio required for analyses such as that shown in Figure 1 is a topic under active investigation (Sun et al. 2014), but preliminary results suggest that a $20 \sigma$ detection is sufficient. We therefore require an rms sensitivity of $5 \mu \mathrm{Jy}_{\text {beam }}{ }^{-1}$ over the entire bandpass $(650-1670 \mathrm{MHz})$. To cover this bandpass with a 500-MHz instantaneous bandwidth, we require two passes over the sky: a survey at $900 \mathrm{MHz}$ with a 55\% fractional bandwidth, and a survey at $1420 \mathrm{MHz}$ with a 35\% fractional bandwidth. For a typical source spectral index $\alpha=-0.7$, this requires an rms of $8.5 \mu \mathrm{Jy}_{\text {beam }}^{-1}$ at $900 \mathrm{MHz}$, and $6.2 \mu \mathrm{Jy} \mathrm{beam}^{-1}$ at $1420 \mathrm{MHz}$. Figure 9 of Braun (2014) presents the sensitivity reached by SKA1-SUR as a function of frequency and resolution, assuming a $30 \%$ fractional bandwidth and 10 hours per pointing. Scaling from these numbers at $1^{\prime \prime}$, we require 4.4 and $1.3 \mathrm{hrs}$ per pointing at 900 and $1420 \mathrm{MHz}$ respectively. For $\approx 1700$ pointings over $30000 \mathrm{deg}^{2}$, the total survey time is around 10000 hours. We note that the $1420-\mathrm{MHz}$ component of this survey will simultaneously provide the data needed for observations of an RM grid (Johnston-Hollitt et al. 2015).

For the case of intervenors along the line of sight ( $\$ 3)$, we motivate our survey on the results of Farnes et al. (2014b), who were able to show a difference in the radio polarisation properties of quasars with and without foreground $\mathrm{Mg}$ II absorbers at 3.5 $\sigma$ significance, using $~ 140$ quasars of which $\sim 40 \%$ have one or more Mg II absorbing systems along the line of sight. For SKA1, we aim to double this sample size in subcategories divided into 10 bins of redshift, 5 bins for absorber equivalent-width, and 5 bins of intervenor impact parameter, for a total of 50000 quasar sightlines. Estimating that quasars are $\sim 10 \%$ of the radio source population at the relevant flux levels in total intensity, we therefore require $\sim 7 \times 10^{5}$ polarised sources in total, or again a sky

\footnotetext{
${ }^{5}$ Almost all these extended polarised sources fall in the redshift range $z \sim 0.3-1$, matching the redshift range covered by SKA1-SUR Band 2 as discussed in $\$ 5.1$ above
} 
density of 23 sources/deg ${ }^{2}$ over $30000 \mathrm{deg}^{2}$. The required rms can be achieved by the same survey as described above. Note that we assume that all 70000 radio-loud quasars will have associated optical spectroscopy from future surveys, which is reasonable given that the required sky density of such spectra $\left(\lesssim 2\right.$ sources/deg $\left.{ }^{2}\right)$ is well below that already available in the northern hemisphere through SDSS ( $\approx 10$ quasar spectra per $\mathrm{deg}^{2}$ ).

The total yield of the proposed survey will be $7 \times 10^{5}$ polarised sources detected at $\geq 20-\sigma$ significance, corresponding to total intensity fluxes $\gtrsim 5-10 \mathrm{mJy}$. We will be able to make use of $\sim 75 \%$ of radio galaxies at these flux levels (Wilman et al. 2008) if we seek to apply rest-frame corrections for redshifts $0.5 \lesssim z \lesssim 3$ (see $\S 5.1$ ).

\section{An Early Science Program for SKA1}

A valuable early science program on broadband polarimetry can be pursued once SKA1 reaches $50 \%$ of its full sensitivity. At this stage, SKA1-SUR will consist of 36 12-metre dishes and 24 15-metre dishes, and will have $\sim 5$ times the survey speed of ASKAP. One of the survey programs intended for ASKAP is the Polarisation Sky Survey of the Universe's Magnetism (POSSUM; Gaensler et al. 2010), which will image $30000 \mathrm{deg}^{2}$ of the polarised sky to a sensitivity of $\sim 10 \mu \mathrm{Jy}_{\text {beam }^{-1}}$ at $10^{\prime \prime}$ resolution and over the frequency range 1.2-1.5 GHz. Early science for SKA1 could consist of a survey with similar sky coverage, angular resolution and sensitivity to that planned for POSSUM, but covering the frequency range 700-1200 MHz. This would require 2-3 months of observing time with the first 50\% of SKA1-SUR. The two data sets would then be combined to provide $700-1500 \mathrm{MHz}$ observations at $\approx 7 \mu \mathrm{Jy}_{\text {beam }}{ }^{-1}$ sensitivity at $10^{\prime \prime}$ resolution.

While such a data set would not have the high angular resolution and large frequency coverage offered by the full set of broadband observations described in $\S 5$, it would provide vital input into the design and approach of these subsequent programs. For example, for studying the interaction of radio lobes with their environments ( $\$ 2)$, the proposed early science program would identify $\sim 10^{4}$ polarised radio lobes that would be spatially resolved at $\approx 10^{\prime \prime}$ resolution and that would show signatures of thermal entrainment. This is $10 \%$ of the full sample size envisaged in $\S 5.3$, which would allow us to identify and study the most prominent cases, and to develop the algorithms and models needed to interpret the larger data sets to follow.

\section{Considerations for SKA2}

The increased sensitivity of SKA2 will obviously allow measurements of effects due to much smaller magnetic field strengths and electron densities in a much larger sample of sources. However, the key point for polarimetry experiments with SKA2 is the increase in the available frequency range, i.e., wide-field survey capability covering 350-1500 MHz. This will allow an extension of the studies described in $\$ 5.1$ out to redshifts $z \sim 3-4$, corresponding to lookback times when we expect substantial evolution in magnetic field geometry and dynamo activity (Arshakian et al. 2009). Over the next 5 years, new results on the polarised sky from POSSUM, WODAN and the JVLA will greatly advance our understanding of all the issues discussed throughout this Chapter, and will help fully define the goals and expected outcomes for SKA2. 


\section{Summary and Conclusions}

We have described a new polarimetric landscape in which a wide range of Faraday effects have now been identified, each of which provides a unique probe of fundamental properties of galaxies. Over relatively narrow fractional bandwidths $(\lesssim 30 \%)$, these effects are either undetectable or highly degenerate. However, by using sensitive spectropolarimetric surveys over broad bandwidths, we can reveal the mechanisms by which supermassive black holes couple to their environments, can measure the properties of ordinary galaxies over a large range in redshift, and can understand how different radio source populations have evolved over the Universe's history. Using SKA1SUR over its full Band 2 frequency range (650-1670 MHz), we can detect polarised emission from the tens of thousands of sources needed to address all these experiments out to redshifts beyond $z \sim 1$. With SKA2, the increased sensitivity and broader frequency coverage will allow us to extend these observations to redshifts $z \sim 3-4$, allowing us to couple existing studies of galaxy evolution and star-formation history to as yet unaddressed questions of how turbulence, ionisation fraction, entrainment efficiency and magnetic field properties have all evolved over cosmic time.

\section{Acknowledgments}

B.M.G. acknowledges the support of the Australian Research Council (ARC) through an Australian Laureate Fellowship (FL100100114), and through the ARC Centre of Excellence for Allsky Astrophysics (CE110001020). We thank the referee for a constructive review of the original manuscript.

\section{References}

Akahori, T., Gaensler, B. M., \& Ryu, D. 2014, ApJ, 790, 123

Andrecut, M., Stil, J. M., \& Taylor, A. R. 2012, AJ, 143, 33

Arshakian, T. G. \& Beck, R. 2011, MNRAS, 418, 2336

Arshakian, T. G., Beck, R., Krause, M., \& Sokoloff, D. 2009, A\&A, 494, 21

Beck, R., Frick, P., Stepanov, R., \& Sokoloff, D. 2012, A\&A, 543, A113

Beck, R. \& Gaensler, B. M. 2004, New Astron. Rev., 48, 1289

Bell, M. R., Junklewitz, H., \& Enßlin, T. A. 2011, A\&A, 535, A85

Bernet, M. L., Miniati, F., \& Lilly, S. J. 2012, ApJ, 761, 144

-. 2013, ApJ, 772, L28

Brandenburg, A. \& Stepanov, R. 2014, ApJ, 786, 91

Braun, R. 2014, SKA1 Level 0 Science Requirements

Brentjens, M. A. \& de Bruyn, A. G. 2005, A\&A, 441, 1217

Carilli, C. \& Rawlings, S. 2004, New Astron. Rev., 48, 979

Farnes, J. S., Gaensler, B. M., \& Carretti, E. 2014a, ApJS, 212, 15

Farnes, J. S., O'Sullivan, S. P., Corrigan, M. E., \& Gaensler, B. M. 2014b, ApJ, 795, 63

Farnsworth, D., Rudnick, L., \& Brown, S. 2011, AJ, 141, 191

Gaensler, B. M. 2004, Key Science Projects for the SKA (SKA Memo Series, No. 44)

Gaensler, B. M., Beck, R., \& Feretti, L. 2004, New Astron. Rev., 48, 1003 
Gaensler, B. M., Landecker, T. L., \& Taylor, A. R. 2010, BAAS, 42, 470.13

Grant, J. K. et al. 2010, ApJ, 714, 1689

Guidetti, D., Laing, R. A., Bridle, A. H., Parma, P., \& Gregorini, L. 2011, MNRAS, 413, 2525

Guidetti, D., Laing, R. A., Croston, J. H., Bridle, A. H., \& Parma, P. 2012, MNRAS, 423, 1335

Hales, C. A., Gaensler, B. M., Norris, R. P., \& Middelberg, E. 2012, MNRAS, 424, 2160

Hales, C. A., Norris, R. P., Gaensler, B. M., \& Middelberg, E. 2014a, MNRAS, 440, 3113

Hales, C. A. et al. 2014b, MNRAS, 441, 2555

Hammond, A. M., Robishaw, T., \& Gaensler, B. M. 2014, ApJS, submitted

Hogg, D. W., Baldry, I. K., Blanton, M. R., \& Eisenstein, D. J. 2012, arXiv:astro-ph/0210394

Horellou, C. \& Fletcher, A. 2014, MNRAS, 441, 2049

Ideguchi, S., Takahashi, K., Akahori, T., Kumazaki, K., \& Ryu, D. 2014, PASJ, 66, 5

Johnston-Hollitt, M. et al. 2015, "Using SKA Rotation Measures to Reveal the Mysteries of the Magnetised Universe" in proc. Advancing Astrophysics with the Square Kilometre Array, PoS(AASKA14)092

Kanekar, N. \& Briggs, F. H. 2004, New Astron. Rev., 48, 1259

Krause, M. et al. 2009, MNRAS, 400, 646

Kronberg, P. P. et al. 2008, ApJ, 676, 70

Kumazaki, K., Akahori, T., Ideguchi, S., Kurayama, T., \& Takahashi, K. 2014, PASJ, 66, 617

Law, C. J. et al. 2011, ApJ, 728, 57

Macquart, J.-P., Ekers, R. D., Feain, I., \& Johnston-Hollitt, M. 2012, ApJ, 750, 139

Morganti, R., Sadler, E. M., \& Curran, S. J. 2015, "Cool Outflows and HI absorbers with SKA" in proc. Advancing Astrophysics with the Square Kilometre Array, PoS(AASKA14)134

Oppermann, N. et al. 2014, A\&A, submitted (arXiv:1404.3701)

-. 2012, A\&A, 542, A93

O'Sullivan, S. P. et al. 2012, MNRAS, 421, 3300

-. 2013, ApJ, 764, 162

Rudnick, L. \& Owen, F. N. 2014, ApJ, 785, 45

Stepanov, R., Arshakian, T. G., Beck, R., Frick, P., \& Krause, M. 2008, A\&A, 480, 45

Stil, J. M., Keller, B. W., George, S. J., \& Taylor, A. R. 2014, ApJ, 787, 99

Sun, X. et al. 2014, AJ, 149, 60

Taylor, A. R. et al. 2007, ApJ, 666, 201

Taylor, A. R., Stil, J. M., \& Sunstrum, C. 2009, ApJ, 702, 1230

Wilman, R. J. et al. 2008, MNRAS, 388, 1335 